\title{
A transcriptional insulator at the imprinted H19/Igf2 locus
}

\author{
Christopher R. Kaffer, Madhulika Srivastava, Kye-Yoon Park, Elizabeth Ives, Sandra Hsieh, \\ Juan Batlle, Alexander Grinberg, Sing-Ping Huang, and Karl Pfeifer ${ }^{1}$
}

Laboratory of Mammalian Genes and Development, National Institute of Child Health and Human Development, National Institutes of Health, Bethesda, Maryland 20892 USA

\begin{abstract}
Igf2 and $\mathrm{H19}$ exhibit parent-of-origin-specific monoallelic expression. $\mathrm{H19}$ is expressed from the maternal chromosome and Igf2 from the paternal. The two genes share enhancer elements and monoallelic expression of both genes is dependent on cis-acting sequences upstream of the $\mathrm{H19}$ promoter. In this work we examine the mechanisms by which this region silences the maternal Igf2 allele and we demonstrate that deletion of this region can result in high levels of activation of both $\mathrm{H19}$ and Igf2 from a single chromosome. Moreover, by inserting this cis element between a promoter and its enhancer at a heterologous position, we demonstrate that the sequences carry both insulator activity and the ability to be stably imprinted. We also characterize the insulator in vitro and show that it is neither enhancer nor promoter specific.
\end{abstract}

[Key Words: Transcription; epigenetics; chromatin; methylation; muscle]

Received March 15, 2000; revised version accepted May 31, 2000.

H19 and Igf2 are two monoallelically expressed genes that are part of a cluster of imprinted genes on the distal end of mouse chromosome 7 (Fig. 1). H19 is expressed only from the maternal chromosome (Bartolomei et al. 1991), whereas Igf2 is generally expressed only from the paternal chromosome (DeChiara et al. 1991). The two genes share complex patterns of developmental and tissue-specific gene expression, suggesting that their transcriptional activation is mechanistically linked. In fact, it has been demonstrated that enhancer elements, at least for endodermal-specific expression, are shared by the two genes (Yoo-Warren 1988; Leighton et al. 1995b). These enhancers lie between 7 and $9 \mathrm{~kb}$ downstream of the $H 19$ promoter and $\sim 100 \mathrm{~kb}$ downstream of the Igf2 promoter, and are capable of directing high levels of transcriptional activation of each gene.

The mechanistic basis for monoallelic expression of H19 has been studied extensively by molecular and genetic approaches. One focus of research has been to detect epigenetic differences in the two chromosomes that might be the actual genomic imprint or that might play a role in maintaining monoallelic expression. Centered at -2.4 and $-3.8 \mathrm{~kb}$ relative to the start of $H 19$ transcription, lie regions showing nuclease hypersensitivity specific to the maternal chromosome (Hark and Tilghman 1998; Szabo et al. 1998; Khosla et al. 1999). These regions of differential hypersensitivity were identified in somatic tissue and are located within a large domain of DNA showing hypermethylation specific to the paternal

${ }^{1}$ Corresponding author.

E-MAIL kpfeifer@helix.nih.gov; FAX (301) 4020543. chromosome (Bartolomei et al. 1993; Brandeis et al. 1993; Ferguson-Smith et al. 1993; Tremblay et al. 1995, 1997; Olek 1997). This differentially methylated region (DMR) is distinctly marked through all stages of development and thus has been considered a good candidate for the actual genomic imprint.

Multiple-copy insertions of $H 19$ transgenes carrying these upstream sequences to $-4 \mathrm{~kb}$ exhibit parent-of-origin-dependent DNA methylation and RNA expression patterns like those at the endogenous locus (Bartolomei et al. 1993; Pfeifer et al. 1996; Elson and Bartolomei 1997; Brenton et al. 1999) However, the same constructs, when present in single copy, are not imprinted. The lack of parent-of-origin-specific expression of single copy transgenes indicates that all of the sequence information required for imprinting is not carried on these constructs.

By directly testing the necessity of the DMR for imprinting the endogenous H19 gene, Thorvaldsen and colleagues deleted the region located between -3.7 and -2.1 $\mathrm{kb}$. Inheritance of the deletion on the paternal chromosome results in activation of the normally silent paternal H19 allele (Thorvaldsen et al. 1998). Analysis of a conditional mutation in which the DMR was removed at varying stages of development demonstrates that the upstream region was required in the germ cells and in early embryonic development to mark the paternal chromosome. However, it was not required late in development to maintain transcriptional silencing of the paternal H19 (Srivastava et al. 2000). Rather, the DMR directed epigenetic changes in the promoter region that were sufficient to silence the paternal H19. Once these epigenetic 


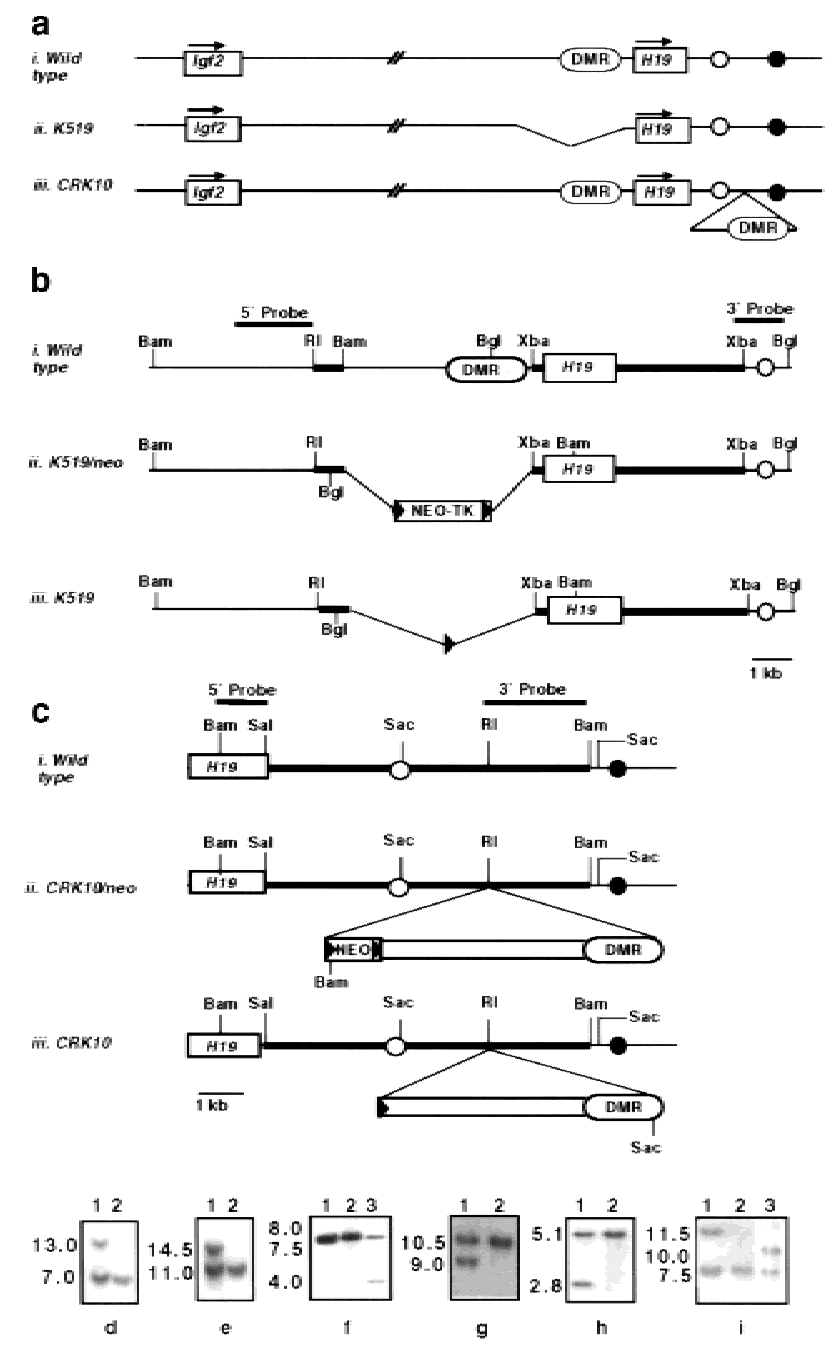

Figure 1. Construction of the $K 519$ allele deleting -10 to -0.8 $\mathrm{kb}$ upstream of the $H 19$ gene and the CRK10 allele inserting these sequences at $+10.7 \mathrm{~kb}$. (a) Summary of the wild-type (i), mutant K519(ii), and CRK10 (iii) chromosomes in the Igf2/H19 region, including transcripts (arrows), shared endodermal enhancers $(\bigcirc)$, skeletal muscle enhancers $(\bullet)$, and sequences hypermethylated specifically on the paternal chromosome (DMR). (b) Strategy for constructing the K519 allele. The wild-type chromosome (i), the targeted K519neo chromosome (ii), and the targeted chromosome K519 after Cre recombinase-mediated excision of the NEO-TK selectable markers (iii) are depicted. (c) Strategy for constructing the CRK10 allele. This mutation inserts the putative insulator between the endodermal and mesodermal enhancers of H19. The wild-type chromosome (i), the targeted CRK10neo chromosome (ii), and the targeted chromosome CRK10 after Cre recombinase-mediated excision of the NEO selectable marker (iii) are depicted. LoxP sites are indicated by arrowheads. $(d-i)$ Southern blot analyses confirmed the structure of the targeted mutations K519neo, K519, CRK10neo, and CRK10 as described in Materials and Methods. (d1,d2) 5' Probe of K519neo and wild-type cells, respectively. (e1,e2) 3' Probe of K519neo and wild-type cells, respectively. (f1-f3) 5' Probe of K519neo, wild-type, and K519 cells, respectively. $(g 1, g 2) 5^{\prime}$ Probe of CRK10neo and wild-type cells, respectively. $(h 1, h 2) 3^{\prime}$ Probe of CRK10neo and wild-type cells, respectively. (i1-i3) 5' Probe of CRK10neo, wild-type, and CRK10 cells, respectively. (Bam) BamHI; (Bgl) BglII; (RI) EcoRI; (Xba) XbaI. changes were made, the upstream region became superfluous.

The mechanism by which the DMR controls monoallelic expression of $I g f 2$, a gene located $90 \mathrm{~kb}$ upstream, is not yet understood. $H 19^{\Delta 13}$, a mutation that removes the $H 19$ gene body and upstream sequences to $-10 \mathrm{~kb}$, resulted in activation of the normally silent maternal Igf2 allele (Leighton et al. 1995). These results were suggestive of a competition between the H19 and Igf2 promoters for their shared enhancers. By this model, the paternal imprint at the DMR somehow acts to shut down the H19 promoter, the otherwise superior competitor, thus permitting enhancer activation of Igf2 transcription. Essentially, this model predicts that the two genes cannot be expressed from a single chromosome. Results from the Thorvaldsen deletion of the DMR, examining expression in liver, were largely consistent with the following model: the activation of the paternal H19 upon paternal inheritance of the deletion was accompanied by decreased expression of the paternal Igf2 (Thorvaldsen et al. 1998). However, a more recent experiment, deleting the $H 19$ promoter on the maternal chromosome did not result in activation of the maternal Igf2 allele as predicted by the competition model (Schmidt et al. 1999). In addition, maternal deletion of the DMR specifically in differentiated tissues caused biallelic expression of $I g f 2$, even though there was no effect on monoallelic expression of H19, indicating that the transcriptional status of Igf2 does not directly reflect the activity of the H19 promoter lying in cis (Srivastava et al. 2000).

An alternate model postulates that silencing of the maternal Igf2 is mediated by the action of an insulator element upstream of the $H 19$ promoter coincident with the DMR and nuclease hypersensitive sites. When active, as on the maternal chromosome, the insulator would function in a position-dependent manner to prevent activation of the Igf2 promoter by the downstream enhancers. On the paternal chromosome, epigenetic modifications would abrogate the insulating activity, permitting activation of the paternal Igf2 promoter. This model does not demand that expression of Igf2 and H19 from a single chromosome be mutually exclusive. It does predict that all enhancer elements will be located downstream of the insulator and that the DMR sequences have insulator activity. The insulator model is consistent with results from the four $\mathrm{H} 19$ deletion mutations described above.

In this work, we address the mechanism for silencing the maternal Igf2 allele. Our work provides direct evidence for the presence of an insulator element upstream of $H 19$ that, when maternally inherited, can suppress interactions between downstream enhancers and upstream promoter elements. Specifically, we characterize a mutation removing sequences between -10 and $-0.8 \mathrm{~kb}$ and demonstrate that, in contrast to predictions of the competition model, coexpression of $H 19$ and Igf2 from a single chromosome is possible. Second, we use transgenes and transfection experiments to map the H19 mesodermal enhancers to a position downstream of the previously mapped endodermal enhancers, a location con- 
sistent with the insulator model. Coincidentally, we identify upstream sequences sufficient to imprint even single-copy $H 19$ transgenes. Third, we directly demonstrate insulator function in the sequences upstream of the H19 promoter by inserting them into a heterologous position in the chromosome. We confirm independently the presence of insulator activity on these sequences using an in vitro assay. Finally, we demonstrate that these upstream sequences carry information that defines their parental origin.

\section{Results}

\section{Deletion of the DMR sequences}

The competition model predicts that activation of the Igf2 promoter is a reflection of the silencing of the cis H19 promoter. Thus, Igf2 and H19 should not be expressed from a single chromosome at the same time. In liver cells, consistent with the competition model, paternal inheritance of a deletion of the DMR results not only in activation of the normally silent paternal H19 allele, but also reduced levels of Igf2 expression (Thorvaldsen et al. 1998). However, Igf2 was also down-regulated in liver upon paternal inheritance of the $H 19^{\Delta 13}$ mutation that removed the entire $H 19$ gene, including the H19 promoter, in which intergenic competition was unlikely. Therefore, we decided to examine the effects of paternal inheritance of a DMR deletion chromosome in additional tissues at various developmental stages to see whether we could identify conditions in which coexpression of H19 and Igf2 at high levels was possible. We used mice that carried a simple deletion of sequences from -10 to $-0.8 \mathrm{~kb}$ (Fig. 1). This deletion, K519, is similar to the Thorvaldsen deletion, but extends $5^{\prime}$ to remove all of the known maternal-specific DNase I hypersensitive sites (Hark and Tilghman 1998; Szabo et al. 1998; Khosla et al. 1999) and all of the upstream sequences required for imprinting of single-copy transgenes (see below).

Paternal inheritance of the K519 mutation led to activation of paternal H19 in all embryonic tissues as determined by in situ hybridization (Fig. 2a,b,c). The relative contribution of the paternal allele was quantitated by single nucleotide primer extension (SNuPE) assay (Kuppuswamy et al. 1991; Singer-Sam et al. 1992). The ability of this assay to quantitate the relative contributions of maternal and paternal alleles to the total H19 and Igf2 RNA levels within a single sample have been established previously (Szabo and Mann 1995; Srivastava et al. 2000). In liver, muscle, heart, and gut, the paternal K519 chromosome contributes $\sim 50 \%$ of the total $H 19$ expression (Fig. 3a; Table 1). The high degree of H19 activation in these tissues was confirmed by Northern analysis of RNA from neonatal pups carrying a paternal K519 chro-
Figure 2. Expression analysis in $\mathrm{K} 519 \mathrm{mu}-$ tant and in H19-BAC1 transgenic e14.5 embryos. Sagittal sections along the midline axis of $\mathrm{H}_{19^{+}} / \mathrm{H}_{1} 9^{+}(a), H 19^{\Delta 13} / \mathrm{H}_{1} 9^{+}(b)$, $H 19^{\Delta 13} / H 19^{K 519}(c)$, and $H 19^{\Delta 13} / H 19^{\Delta 13}$, H19-BAC1 transgenic $(d)$ mice were hybridized in situ to ${ }^{33} \mathrm{P}$-labeled riboprobes antisense to $H 19$. The $H 19^{\Delta 13}$ deletion removes the entire $H 19$ gene. Thus, $a$ and $b$ represent positive and negative controls, respectively. (c) Expression from the paternal K519 chromosome. (d) Expression from a maternally inherited H19-BAC1 transgene. Similarly, sagittal sections of a wild-type embryo $(e)$ and an embryo inheriting the K519 mutation paternally $(f)$ were hybridized to riboprobes antisense to Igf2. Blue and orange regions depict the embryonic tissue and hybridization signals, respectively.
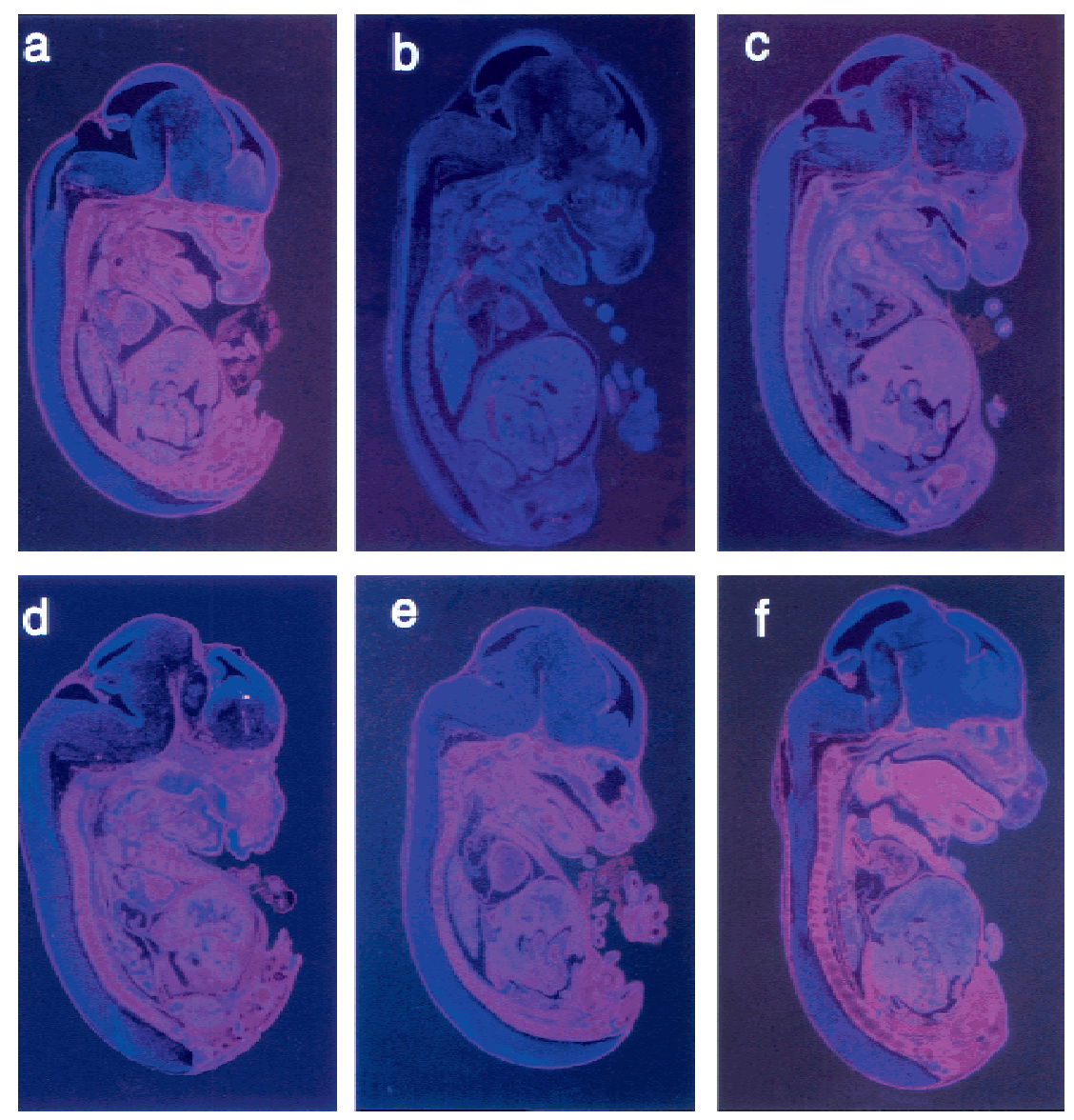
mosome and a maternal chromosome with the $H 19^{\Delta 13}$ mutation (Fig. 3c). In other tissues, the activation of $H 19$ was reduced compared with that seen from wild-type maternal chromosomes (data not shown), consistent with previous reports (Thorvaldsen et al. 1998).

Transcription of the paternal $\mathrm{H} 19$ allele did not always lead to a concomitant loss of Igf2 expression as seen by in situ hybridization (Fig. 2e,f). Northern hybridization confirmed that levels of Igf2 from the paternal K519 chromosome were like those noted from wild type in skeletal muscle and gut (Fig. 3c) and in heart, kidney, brain, and lung (data not shown). Liver RNA was reduced in total Igf2 levels as reported previously (Thorvaldsen et al. 1998). We conclude that activation of paternal H19

a.

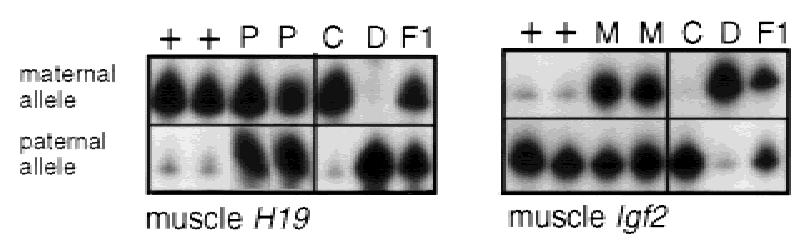

c.

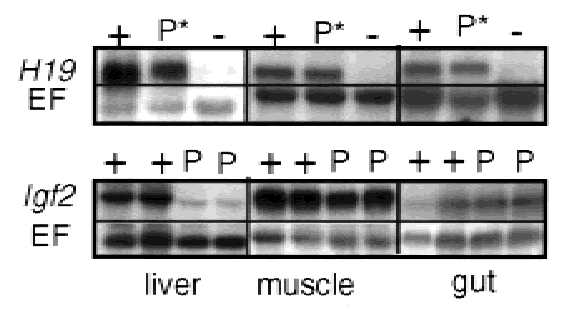

d.

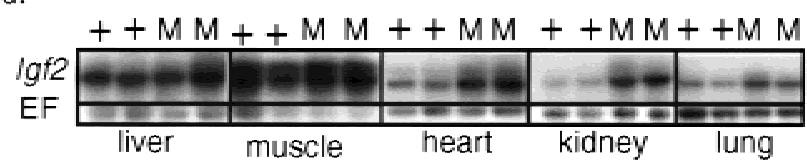

Figure 3. Expression of $H 19$ and $I g f 2$ in $K 519$ neonates. Allelespecific expression patterns of $H 19(a)$ and of $I g f 2(b)$ were analyzed by SNuPE assay. (a) H19 expression is analyzed in neonates carrying a mutated paternal allele $(\mathrm{P})$ on a domesticus chromosome and a maternally inherited wild-type castaneous allele. Control littermates (+) have inherited a wild-type domesticus allele paternally and a wild-type castaneous allele maternally. (b) Igf2 expression is analyzed in neonates carrying a mutated maternal allele $(\mathrm{M})$ on a domesticus chromosome and a paternally inherited wild-type castaneous allele. Control littermates $(+)$ have maternally inherited a wild-type domesticus allele and paternally inherited a wild-type castaneous allele. For both $a$ and $b$, lanes $C, D$, and $F 1$ depict results for a pure castaneous DNA template, a pure domesticus DNA template, and DNA from a castaneous $\mathrm{x}$ domesticus $\mathrm{F} 1$ animal, which therefore represents a 50:50 mix of the two DNAs. Total RNA levels for $H 19$ and Igf2 were analyzed by Northern blot upon paternal inheritance $(c)$ or upon maternal inheritance $(d)$ of the $K 519$ allele. The identity of the probes is indicated at left. The level of elongation factor (EF) RNA was assayed as a control. $(+) \mathrm{H}^{19^{+}} /$

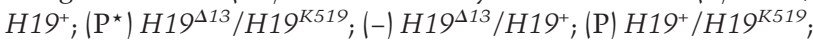
(M) $H 19^{K 519} / \mathrm{H}^{2} 9^{+}$.
Table 1. The effect of the K519 mutation on allele specific expression

\begin{tabular}{|c|c|c|c|c|}
\hline \multirow[b]{2}{*}{ Tissue } & \multicolumn{2}{|c|}{$\begin{array}{l}\text { Contribution of } \\
\text { the paternal } \\
\text { chromosome to } \\
\text { total H19 mRNA }\end{array}$} & \multicolumn{2}{|c|}{$\begin{array}{l}\text { Contribution of } \\
\text { the maternal } \\
\text { chromosome to } \\
\text { total Igf2 mRNA }\end{array}$} \\
\hline & $\begin{array}{c}H 19^{+} / \\
H 19^{K 519}\end{array}$ & $\begin{array}{c}H 19^{+} / \\
H 19^{+}\end{array}$ & $\begin{array}{c}H 19^{\mathrm{K} 519} / \\
\mathrm{H} 19^{+}\end{array}$ & $\begin{array}{c}{\mathrm{H} 19^{+} /} \\
\mathrm{H} 19^{+}\end{array}$ \\
\hline Liver & $46 \pm 2$ & $10 \pm 4$ & $20 \pm 3$ & $<1$ \\
\hline Muscle & $43 \pm 8$ & $5 \pm 2$ & $40 \pm 4$ & $4 \pm 1$ \\
\hline Heart & $44 \pm 4$ & $3 \pm 4$ & $49 \pm 3$ & $4 \pm 3$ \\
\hline Kidney & $26 \pm 8$ & $7 \pm 2$ & $47 \pm 5$ & $<1$ \\
\hline Lung & $27 \pm 4$ & $4 \pm 4$ & $37 \pm 3$ & $4 \pm 1$ \\
\hline Gut & $58 \pm 6$ & $10 \pm 3$ & $40 \pm 4$ & $2 \pm 1$ \\
\hline Brain & $61 \pm 5$ & $10 \pm 3$ & $61 \pm 5$ & $52 \pm 18$ \\
\hline
\end{tabular}

Samples were analyzed by SNuPE assay as described in Fig. 3. For the $H 19$ assay, control experiments indicate that pure DNA samples give a background of $10 \pm 3 \%$ due to misincorporation during the SNuPE protocol. For the Igf2 assay, the background is estimated at $6 \pm 4 \%$. The numbers shown are not corrected for this background.

was not at the expense of paternal Igf2 expression and our results, therefore, are not well explained by the competition model.

When maternally inherited, the K519 mutation led to activation of the normally silent maternal Igf2 promoter in all tissues tested. Total Igf2 RNA increased approximately twofold in all non-hepatic tissues from mutant neonates (Fig. 3d), consistent with activation of both the mutant maternal and the wild-type paternal Igf2 alleles. SNuPE assays confirm that the maternal chromosome contributed $\sim 50 \%$ of the total Igf2 RNA in all tissues except the liver (Fig. 3b; Table I). These results were consistent with the presence of a transcriptional insulator element upstream of H19. Our subsequent experiments were therefore directed toward directly testing the insulator model for monoallelic expression of Igf2.

\section{Mapping mesodermal enhancers of $\mathrm{H} 19$}

To prevent blocking of H19 expression from the maternal chromosome, in which the putative insulator is predicted to be active, the insulator model demands that all H19 enhancer elements be located 3' of the DMR region. Consistent with the model, endodermal enhancers are located between +7 and $+9.5 \mathrm{~kb}$ (Leighton et al. 1995b; Yoo-Warren et al. 1988). However, the exact location of the mesodermal enhancers has been unknown. Previous transgenic experiments have mapped skeletal muscle enhancers to either upstream of $-4 \mathrm{~kb}$ or between +12.5 and +36 kb (Brunkow and Tilghman 1991; Pfeifer et al. 1996; Ainscough et al. 1997). Likewise, these same experiments indicate that enhancers responsible for cardiac muscle expression lie either downstream of $+36 \mathrm{~kb}$ or upstream of the Igf2 gene. To help distinguish between these possible locations, we generated transgenic lines using a bacterial artificial chromosome (BAC) clone that spans from -7 to about $+130 \mathrm{~kb}$. We analyzed two lines 


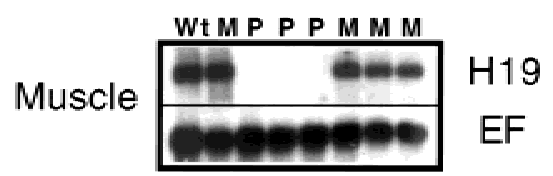

Figure 4. Expression of H19 in skeletal muscle from the H19BAC1 transgene. RNA was prepared from tissues dissected from day 5 pups carrying the H19-BAC1 transgene in an $H 19^{\Delta 13}$ / $\mathrm{H}_{19^{+}}$background. RNA was assayed by Northern analysis comparing pups in which the transgene was inherited maternally (M) or paternally $(\mathrm{P})$ with wild-type $(\mathrm{Wt})$ littermates. Results for one of two independent lines are depicted.

in which a single copy of the entire BAC clone was inserted at sites unlinked to the H19/Igf2 locus (data not shown). In situ hybridization experiments demonstrate a pattern of expression from the H19-BAC1 transgene that is highly similar to that of the endogenous locus (Fig. 2d). Furthermore, Northern analyses of RNA prepared from skeletal muscle of day 5 pups confirm that the BAC transgene contains sequences sufficient for this mesodermal expression of H19 (Fig. 4). Taken together with the previously published transgenic results and the continued skeletal muscle expression in the K519 deletion mutant, these BAC transgenic analyses place the skeletal muscle enhancers between +12 and $+36 \mathrm{~Kb}$. This location is consistent with predictions of the boundary model.

As seen in Figure 4, H19 is expressed from the BAC transgene only upon maternal inheritance. Maternal specific expression was noted in all of the following tissues tested: liver, heart, lung, and kidney. Thus we conclude that upstream sequences to $-7.0 \mathrm{~kb}$ are sufficient for consistent imprinting of even single-copy H19 transgenes.

Our in situ analyses demonstrate expression of the BAC transgene in embryonic cardiac tissue (Fig. 2). These results were confirmed by Northern analysis of
RNAs extracted from neonatal hearts (data not shown). These results, together with previous transgenic analyses (Ainscough et al. 2000), indicate that the cardiac enhancers lie between +36 and $+130 \mathrm{~kb}$. However, we did note that H19-BAC1 expression in heart and in kidney was reduced about three- to fourfold compared with endogenous expression (data not shown).

\section{In vitro mapping of skeletal muscle enhancers}

To confirm the presence of skeletal muscle enhancers in the downstream region and to define their location more precisely, in vitro transfection analysis was performed in two muscle cell lines. Mouse C2C12 and Sol8 cell lines have provided an excellent system for studying musclespecific gene expression (Calvo et al. 1999). Differentiation of these myoblast cell lines into myotubes can be accomplished by the transfer of cells into medium containing reduced levels of serum supplement and results in high-level expression of H19 RNA (data not shown). DNA fragments spanning the +10 to $+42 \mathrm{~kb}$ downstream of the $H 19$ gene were cloned into the multiple cloning site of a reporter vector, $\mathrm{pBgal}$-Promoter. This vector carries an SV4O promoter inserted just upstream of the Escherichia coli lacZ gene. We assayed expression of the lacZ gene in pools of stably transfected cell lines as described in the Materials and Methods section. These results, summarized in Figure 5, show that the insert on Constructs 6 and 7 was able to increase expression from the SV40 promoter by $\sim 400$ - to 500 -fold. Construct 8 , with an insert partially overlapping that of Constructs 6 and 7 , showed a relatively very small enhancement of $\sim 30$-fold. All other constructs demonstrated essentially background levels of gene activation. Given the lack of enhancement from Constructs 4, 5, 9, and even 8, we conclude that the skeletal muscle enhancers for H19
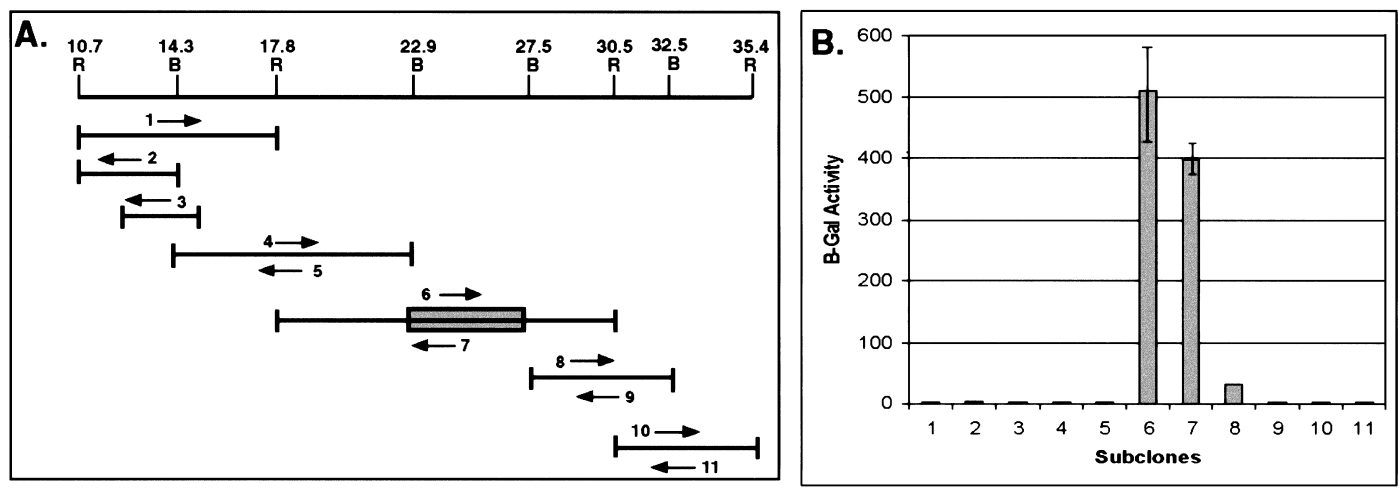

Figure 5. $\beta$-Galactosidase activity from stable transfection of the Sol8 cell line. $(a)$ DNA fragments spanning the +10 to $+35.5-\mathrm{kb}$ region downstream of $H 19$ were cloned into the multiple cloning site of the reporter vector, pßgal-Promoter. This vector carries an SV40 promoter inserted upstream of the lacZ gene. The top line depicts an EcoRI (R) and partial BamHI (B) map of the region. The subcloned fragments are indicated below along with the construct number. The arrow indicates the fragment orientation. $(b)$ Sol 8 myoblasts were cotransfected with the reporter constructs and pTK-Hyg (Clontech) and resistant colonies were selected in hygromycin B. Resistant cells were replated and grown $72 \mathrm{hr}$ in differentiation medium to induce myotube formation. Cell lysates were assayed for $\beta$-galactosidase activity with enzyme activity normalized to total protein levels. The levels of induction relative to that generated by an enhancerless control construct are depicted. 
centers in the sequences between $\sim 22$ and $28 \mathrm{~kb}$ downstream of the promoter (Fig. 5).

We mapped enhancer activity to this same interval using transient transfection of both Sol8 and C2C12 cell lines. In both cell lines, Constructs 6 and 7 were able to enhance expression of the $S V 40$ promoter $\sim 10$-fold (data not shown). No enhancer activity was detected in this assay system using Construct 8. Thus, in both C2C12 and Sol8 cell lines, and measured by both transient and stable transfection, we map skeletal muscle enhancer activity to DNA sequences found in Constructs 6 and 7.

\section{Effect of the CRK10 insertion on H19 expression}

To test directly the ability of the upstream element to prevent enhancer-promoter interactions, we generated a mutation, CRK10, in which the potential insulator element was inserted between the endodermal and the skeletal muscle enhancers located downstream of H19 (Fig. 1). If, in fact, the region acts as an insulator, its effect on transcription will be position dependent. Endodermal enhancers will remain free to interact with the H19 promoter. Skeletal muscle enhancers now separated from the promoter by the insulator will be prevented from activating $H 19$ transcription. The maternally inherited mutant allele will thus express H19 in tissues of endodermal origin but not in tissues of mesodermal origin. RNA from pups maternally inheriting the CRK10 mutation was prepared from liver and skeletal muscle. H19 expression in livers from mutant mice was equal to that seen in wild-type mice. In contrast, skeletal muscle expression of $H 19$ in mutant pups is drastically reduced when compared with wild-type littermates, demonstrating that this element acts in a position-dependent manner to prevent enhancer activity (Fig. 6).

Our experiment cannot directly exclude the possibility that transcription in muscle is reduced due to the increase in distance of the enhancers to the H19 promoter. However, enhancers are known to work over large distances. The endodermal enhancers, for example, direct high levels of expression on both H19 and Igf2 promoters that are 9 and $100 \mathrm{~kb}$ away, respectively. The skeletal muscle enhancers are $>20 \mathrm{~kb}$ distant from the H19 promoter. Although not yet demonstrated experimentally, we expect, on the basis of the nearly identical

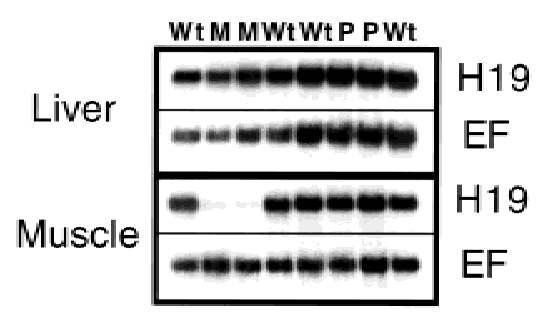

Figure 6. The effect of the CRK10 mutation on H19 transcription in endodermal and mesodermal tissues. H19 RNA levels in skeletal muscle and in liver from day 5 pups inheriting the CRK10 insertion maternally $(\mathrm{M})$ or paternally $(\mathrm{P})$ were compared with wild-type littermates $(\mathrm{Wt})$. expression patterns of Igf2 and H19 in tissues of both endodermal and mesodermal origins, that Igf2 also shares the same mesodermal enhancers with H19 (see also Ainscough et al. 2000). We noted no changes in the levels of Igf2 expression upon paternal inheritance of the CRK10 insertion (C.R. Kaffer and K. Pfeifer, unpubl.).

\section{In vitro demonstration of insulator activity}

We used a colony assay (Chung et al. 1993) to test independently whether DMR-containing sequences could insulate the H19 promoter from the H19 endodermal enhancers. Constructs in which potential insulators were inserted between the $H 19$ endodermal enhancers and the H19 promoter (-800 to $+6 \mathrm{bp})$ were transfected into the human hepatoma cell line, Hep3B, generating cell lines with multicopy insertions of the DNA constructs. In all constructs, the neomycin resistance gene was fused to the H19 promoter so that activation of the promoter could be assayed by counting the number of G418-resistant colonies. If the DMR sequences contain insulator elements, we expected to see a reduction in the number of resistant colonies toward the level noted for control constructs that have no enhancer elements.

When the 4.7-kb XbaI-XbaI fragment covering from -5.5 to $-0.8 \mathrm{~kb}$ of the $H 19$ upstream region was inserted on both sides of the endodermal enhancer (pKP-4), the number of G418-resistant colonies was, in fact, reduced to below those seen in constructs in which no enhancer at all was present (Fig. 7). The same fragment, when inserted only upstream of the enhancer, had no effect on the number of colonies (pKP-3). Thus, its ability to silence the $H 19$ promoter appears to be position dependent. A smaller BglII-BglII fragment carrying sequences between -4.3 and $-1.9 \mathrm{~kb}$ upstream of the H19 start site also showed strong insulator activity (pKP-5).

Next, we tested whether the activity was enhancer specific by assaying constructs in which the SV4O enhancer replaced the $H 19$ endodermal enhancer elements. This assay system was also advantageous because the difference in colony numbers noted with and without enhancer activity was much greater (Fig. 7B). Again in this system, even the 2.4-kb BglII-BglII fragment showed significant insulator activity. The decreased number of colonies was not due to an increased distance between the promoter and the enhancer, as we observed no such decrease when we tested a nonspecific $2.3-\mathrm{kb}$ genomic fragment in the same assay.

These results confirm that there is position-dependent insulator activity in sequences from the upstream region of the $H 19$ gene. This activity is enhancer nonspecific. The activity is also species nonspecific in that the mouse sequences can function as insulators in a human cell line. The smallest fragment we have successfully tested for insulator function includes the DMR and the sites of the maternal-specific nuclease hypersensitivity.

\section{Methylation analysis of the CRK10 allele}

In its normal location upstream of H19, the insulator element sequences are hypermethylated on the paternal 
A

Relative colonies

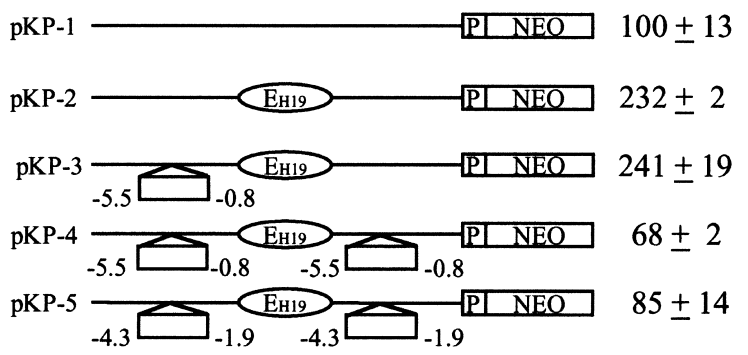

B

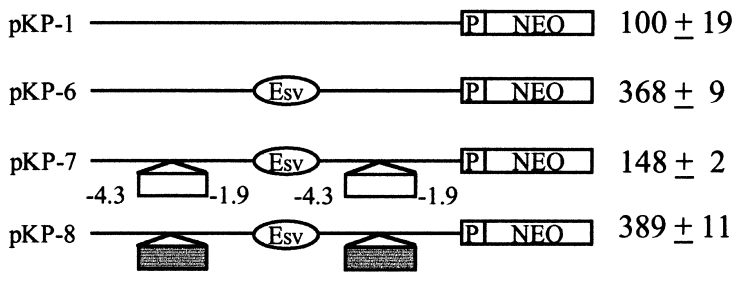

Figure 7. The upstream region of the mouse H19 gene insulates the H19-neomycin resistance gene fusion from enhancer activation. The human hepatoma cell line, Hep3B, was stably transfected with the indicated constructs and grown in medium containing G418. The relative number of resistant colonies is depicted with expression from plasmid pKP-1 (promoter only) set at 100. $\left(\mathrm{E}_{H 19}\right) 2.5-\mathrm{kb} \mathrm{XbaI-XbaI-BgIII} \mathrm{fragment} \mathrm{from} \mathrm{down-}$ stream of $H 19$ that carries the endodermal enhancer elements; $\left(\mathrm{E}_{\mathrm{SV}}\right)$ 256-bp fragment carrying the $S V 40$ enhancer; (P) 800-bp $\mathrm{XbaI}$-DraIII fragment carrying the $\mathrm{H} 19$ promoter; (NEO) neomycin resistance gene. (Rectangles) The insertion of potential insulator elements with the endogenous location of these elements noted. (Filled rectangles) The control fragments from downstream of the $\mathrm{H} 19$ gene. The drawing is not to scale but plasmids are described in detail in Materials and Methods.

chromosome and are essentially unmethylated on the maternal chromosome. To determine whether the inserted element contained sequences sufficient for this imprint, genomic DNAs extracted from the livers of CRK10 mice and their wild-type littermates were subjected to restriction digestion with methylation-sensitive enzymes. When the mutation is inherited from the mother, our displaced insulator, seen as a 3.0-kb EcoRI fragment, is completely sensitive to digestion with ClaI and HhaI (Fig. 8) or with HpaII (data not shown), indicating that the element is unmethylated at these sites. When inherited from the father, however, the inserted element is completely resistant to digestion with these same enzymes, indicating that the sequences are methylated at these sites. We conclude that the inserted element contains the information that is sufficient for marking the H19/Igf2 region as paternal or as maternal in origin.

\section{Discussion}

We provide evidence that maternal silencing of Igf2 is a consequence of the presence of a cis-acting insulator el- ement that acts in a position-dependent manner to prevent enhancers from interacting with the maternal Igf2 promoter. We first show that a deletion of a $9-\mathrm{kb}$ region upstream of the $\mathrm{H} 19$ promoter permits coexpression of H19 and Igf2 from a single chromosome, a result incongruent with the competition model. We then map mesodermal enhancer elements to sequences downstream of the $\mathrm{H} 19$ gene, a position consistent with determination of Igf2 monoallelism via a maternal-specific insulator. Third, we directly demonstrate position-dependent insulator function by inserting the region containing the putative insulator between the liver and skeletal muscleenhancer elements located downstream of H19. In vitro analyses independently confirm the presence of insulator activity and provide additional evidence that loss of enhancer function is not due to changes in the distance between the promoter and enhancer elements. Finally, we demonstrate that this region contains sufficient information to mark its own parental origin. When paternally inherited, the inserted insulator sequences are hypermethylated just as they are at their normal location. We presume that this DNA methylation inactivates the endogenous insulator function and thus permits Igf2 expression from the wild-type paternal chromosome.

Tissue-specific expression of $H 19$ is determined by at least three independent enhancer elements. Expression in endodermal tissues and in skeletal muscle is directed by cis-acting elements centered $\sim 8$ and $25 \mathrm{~kb}$, respectively, downstream of the $H 19$ promoter. Our results also indicated that cardiac muscle enhancers lie down-
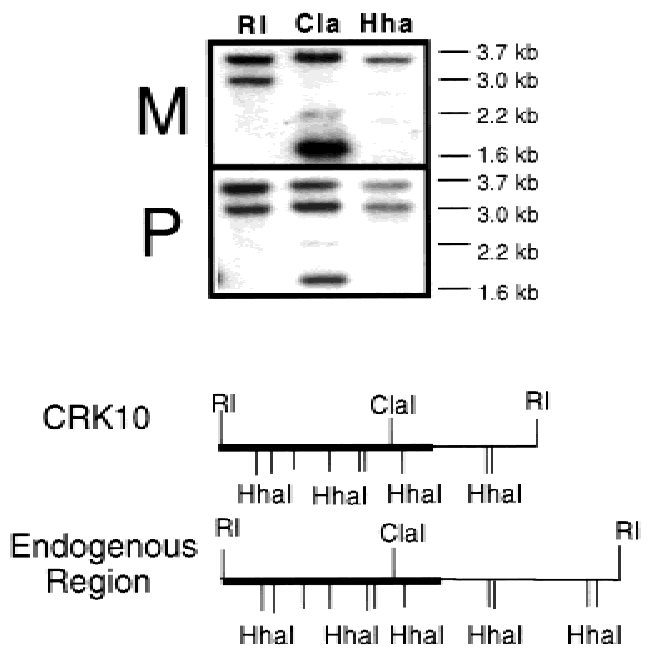

Figure 8. CpG methylation of the inserted element in the CRK10 mutation. Progeny positive for the CRK10 insertional mutation carry three putative insulator regions, two endogenous insulators, maternal and paternal, and one inserted insulator, maternal or paternal dependent upon the cross used to generate the pup. Digestion with EcoRI and hybridizing with a 1.7-kb EcoRI-HindIII probe containing sequences from -3.9 to $-2.2 \mathrm{~kb}$ reveals a $3.7-\mathrm{kb}$ fragment representing the endogenous sequences and a $3.0-\mathrm{kb}$ fragment derived from the inserted element. A restriction map of the endogenous $3.7-\mathrm{kb}$ and inserted 3.0-kb EcoRI fragments is depicted with the probe shown as a thickened line. 
stream of $+35 \mathrm{~kb}$. It has been demonstrated that Igf2 shares the endodermal enhancers (Leighton et al. 1995). We are currently testing the proposition that the other enhancer elements are also shared. On the basis of transgenic analysis (Ainscough et al. 1997, 2000) and on the matched patterns of tissue-specific and temporally regulated patterns of gene expression, we expect that the elements are shared. In that case, activation of the Igf2 promoter is dependent upon cis elements that lie between 110 and $>135 \mathrm{~kb}$ downstream.

Because enhancers can often work across long distances and thus have the potential to affect many different promoters, it seems necessary for the cell to have the ability to limit enhancer accessibility and prevent promiscuous gene expression. Over the past few years, novel regulatory elements, called insulators, have been identified, which restrict access of enhancers to distal promoters and thus do provide a mechanism to stop inappropriate enhancer activity (for reviews, see Bell and Felsenfeld 1999; Sun and Elgin 1999). Insulator elements were first described in Drosophila. Scs and scs' elements were identified as chromatin boundary elements that flank two hsp70 genes (Udvardy et al. 1985). The gypsy retrotransposon carries an insulator element that is responsible for the mutant phenotypes associated with insertion of the transposon (Corces and Geyer 1991). Both gypsy and scs/scs' elements are able to block enhancers in a position-dependent manner: They prevent promoters from being activated when placed between the promoters and their enhancers (Geyer and Corces 1992; Kellum and Schedl 1992). They are also able to insulate transgenes against both positive and negative position effects (Kellum and Schedl 1991; Roseman et al. 1993). In vertebrates, regulation of the chicken $\beta$-globin locus by insulators is well established (Chung et al. 1993). The $\beta$-globin insulator, like the Drosophila elements, can prevent enhancer-promoter interactions in a position-dependent manner and can provide position independence to transgenes. It has been suggested that the insulator may play a role in chromatin organization, organizing the switch from heterochromatin to euchromatin, perhaps through attracting proteins that regulate histone acetylation (Hebbes et al. 1994).

Deleting sequences carrying the DMR and the maternal-specific hypersensitive sites on the maternal chromosome causes full activation of the maternal Igf2 promoter. To determine whether the region upstream of $H 19$ has insulator function, we generated mice carrying an insertion of this region between the endodermal and mesodermal enhancers located downstream of the H19 promoter. The insertion was made at $+10.7 \mathrm{~kb}$, a site at which insertion of a $N e o R$ gene does not interfere with H19 expression in skeletal muscle (Leighton et al. 1995b). As predicted by the insulator model, CRK10 mice carrying the insertion exhibited drastically reduced levels of H19 in skeletal muscle. In contrast, endodermal expression of H19 in mutant pups was essentially normal, indicating that the insertion did not show a generalized silencing of the H19 promoter. Thus, the inserted element does not act in a nonspecific way to silence the
H19 promoter. Rather, the effect is enhancer specific, only expression dependent upon enhancers that are separated from the promoter by the insulator element is blocked. We believe that the upstream element shows a similar position-dependent insulator activity at its endogenous location, and that this activity is responsible for preventing activation of the maternal Igf2 promoter.

We independently assayed the upstream element for insulator activity using a colony assay developed to examine insulator function of the chicken $\beta$-globin locus (Chung et al. 1993) and confirmed the ability of the upstream element to suppress promoter-enhancer interactions in a position-dependent manner. Using this assay, we were able to define a $2.4-\mathrm{kb}$ region containing insulator function. The ability of the insulator to function is not enhancer specific, as it was able to block activation of the H19 promoter by both the SV4O enhancer and by H19 endodermal-specific enhancer elements. Likewise, we surmise that the insulator is not promoter specific, because it works on the $H 19$ promoter and, in its normal location upstream of $H 19$, on the Igf2 promoter. Finally, the in vitro assay system allowed us to demonstrate directly that loss of enhancer activation is not explained by the increased distance between the enhancer and promoter elements that is necessitated by our assay systems.

The colony assay alone cannot rule out that the insulator may also show some silencing activity. The ability of the DMR to act as a silencer when assayed by use of constructs similar to those tested in vitro in this study was demonstrated previously in Drosophila (Lyko et al. 1997). Using constructs in which expression was driven by the H19 endodermal enhancers (Fig. 7A), we noted that presence of the insulator actually reduced the number of G418 resistant clones to $\sim 75 \%$ of that seen with the promoter alone. In fact, we postulate that DMR, in its normal function, does act to unidirectionally silence the $H 19$ promoter via the direction of epigenetic modifications of the promoter postnatally. However, clear evidence that the DMR does carry sequences that do function as insulators comes from the in vivo analysis of mice maternally inheriting the CRK10 insertion. The concomitant activation of the H19 promoter in liver and the lack of activation in skeletal muscle is inconsistent with silencing the $H 19$ promoter by the CRK10 insertion. Thus, in vivo analyses demonstrate that a biologically fundamental function of the DMR is its action as a transcriptional insulator.

Southern analysis of the CRK10 mutation shows identical patterns of methylation at the inserted insulator compared with that seen at the endogenous element. When displaced, it remains unmethylated on the maternal allele, but methylated on the paternal allele, demonstrating that the region contains sufficient information to stably maintain a genomic imprint. We presume that this imprint or the epigenetic changes it directs during development results in inactivation of the insulator function at its normal location, thereby allowing expression of paternal Igf2. The major significance of our results relative to insulator biology is this indication that 
the insulator itself is subject to regulation. In the case of Igf2, the regulation is via the epigenetic modifications induced by the paternal imprint. For other genes, one could anticipate that the modification be developmental or tissue specific, and thus would contribute to regulated gene expression.

The molecular mechanisms for insulator activity are not yet clear, and no current model explains all the experimental data (for reviews, see Bell and Felsenfeld 1999; Sun and Elgin 1999). In one model, proteins associated with the insulator interact with proteins associated with the enhancer and interrupt the ability of the latter to activate proteins interacting with the promoter. At its endogenous location upstream of the H19 promoter, the Igf2 insulator is preventing activation of the maternal Igf2 gene by enhancers that are clearly functional in that they are directing high-level expression of the maternal $H 19$ allele. Thus, as demonstrated previously in transgenic studies of gypsy function, insulators do not actually inactivate enhancers, but only block their motion directionally (Cai and Levine 1995; Scott and Geyer 1995). In a second broad category of models, insulators play a role in higher-order chromatin organization. Any such model for insulator activity at the H19/ Igf2 locus must take into account the ability of the insulator to act upon enhancers and promoters that are $>30$ and $80 \mathrm{~kb}$ distant, respectively (see Bell and Felsenfeld 1999|. Our results agree with a model that assumes that the enhancer is the origin of a progressive signal that must move in cis toward the promoter. However, the nature of such a signal is enigmatic.

In summary, our results support a model in which parental-specific expression of the H19 and Igf2 genes occurs via a shared parental imprint of the DMR region, but in which transcriptional silencing of the paternal H19 and maternal Igf2 alleles occurs via fundamentally different mechanisms. The DMR is required only temporarily during development to establish silencing of the paternal H19 promoter (Srivastava et al. 2000). During development, the DMR directs epigenetic changes in the H19 promoter and gene that are the direct cause of transcriptional silencing. In contrast, presence of the DMR is continually required for silencing of the maternal Igf2 allele (Srivastava et al. 2000), consistent with sequences in the DMR region acting as a transcriptional insulator that blocks enhancer activation of promoters in a position-dependent manner. Both the maternal and paternal Igf2 promoters are thus potentially functional, and only the lack of enhancement on the maternal chromosome prevents transcription of the maternal allele. This view of gene regulation at Igf2 was actually suggested by very early experiments demonstrating that whereas the H19 promoter was differentially accessible to nuclease digestion on the paternal and maternal chromosomes (Bartolomei et al. 1993; Ferguson-Smith et al. 1993), the Igf2 promoter behaved equivalently on both chromosomes (Sasaki et al. 1992). Similarly, moving the endodermal enhancers to a position upstream of the insulator allowed biallelic expression of Igf2 (Webber et al. 1998).

Regulation of expression of $\mathrm{H} 19$ and of Igf2 has been studied extensively by a number of laboratories as a useful model system for understanding the molecular basis of genomic imprinting and the consequent monoallelic expression. As recent experiments demonstrate, the basis of monoallelic expression of the two genes is quite distinct, the lesson of the model system might be that there is no single model to explain parental-specific expression. The H19 and Igf2 genes share enhancer elements, although it is not clear that this is pertinent to regulatory mechanisms for either gene. Considerable experimental evidence now indicates that competition for the shared enhancers is not the basis for the reciprocal pattern of monoallelic expression of $H 19$ and Igf2 in that monoallelic expression of each gene can be maintained independently of the other (Schmidt et al. 1999; Srivastava et al. 2000; this work). The most parsimonious mechanism for imprinting at this locus posits that the two genes share a common imprinting element. Strictly speaking, however, this fact remains to be established experimentally. Whether sequences that function as the imprinting box for $H 19$ and Igf2 are identical, and whether the insulator and the actual imprinting box are distinct, and the molecular mechanisms by which the imprint inactivates the insulator and the $H 19$ promoter all remain to be established.

\section{Materials and methods}

\section{Generation of K519 and CRK10 mutant mice}

The targeting vectors were linearized and transfected into ES cells. To identify K519neo clones, DNAs were digested with BamHI and probed with a $2.2-\mathrm{kb}$ PstI-EcoRI probe from just outside of the $5^{\prime}$ flank to identify a $7-\mathrm{kb}$ fragment in wild-type cells and an additional 13-kb fragment in targeted clones. Likewise, digestion with $B g I I I$ and probing with a $1.8-\mathrm{kb} X b a \mathrm{I}-\mathrm{XbaI}-$ $B g$ III probe from just beyond the $3^{\prime}$ flank results in an $11-\mathrm{kb}$ fragment in wild-type cells and an additional 14.5-kb fragment in targeted clones. Two independent clones were each transiently transfected with pBS185, which contains the Cre recombinase driven by the HCMV promoter/enhancer (Sauer and Henderson 1990). Successful excision of the NEO-TK sequences was assayed by identification of a 4.0-kb $\mathrm{XbaI}$ fragment using the 5' probe described above. The wild-type and K519neo alleles show 8.0 - and $7.5-\mathrm{kb}$ bands, respectively. In addition, the region across the deletion was amplified and its DNA sequence confirmed. Positive cell lines were introduced into C57/BL6-J blastocysts to generate chimeric founder mice.

To identify CRK10neo clones, DNAs were digested with $B a m H I$ and probed with a $1.3-\mathrm{kb}$ BamHI-SalI fragment from just beyond the $5^{\prime}$ flank to identify a 10.5 -kb fragment in wildtype cells and an additional $9.0-\mathrm{kb}$ band in targeted lines. Likewise, digestion with SacI and probing with a $2.2-\mathrm{kb}$ BamHIEcoRI fragment results in a $5.1-\mathrm{kb}$ fragment in wild-type cells and an additional $2.8-\mathrm{kb}$ band in targeted lines. Two lines were used to generate chimeric founders as above. Founder males were mated with mice carrying an EIIa-cre transgene (Lasko et al. 1996) to excise the NEO gene sequences. Successful excision of the NeoR gene was assayed by digestion of genomic DNAs with HindIII and hybridization to the 5' probe used to screen for CRK10neo. Wild-type, CRK10neo, and CRK10 alleles generate 7.5-, 11.5-, and 10.0-kb bands, respectively.

For both K519 and CRK10 constructs, the targeting vector 
included the diptheria toxin A chain gene for negative selection (McCarrick et al. 1993).

Mice carrying the K519 or the CRK10 mutations were crossed with Dis7CAS mice or with $H 19^{\Delta 13} / H 19^{\Delta 13}$ mice as described in the text. Dis7CAS mice are mostly domesticus, but are homozygous castaneus across the H19/Igf2 locus (Gould and Pfeifer 1998).

\section{Isolation and characterization of H19-BAC}

H19-BAC1 was identified in the Genome Systems Release I Library using a 1.7-kb EcoRI-HindIII probe spanning the DMR element upstream of the $H 19$ gene. Southern blot analyses and DNA sequencing demonstrated that the BAC insert contained sequences up to the -7-kb HindIII site. The size of the NotI fragment containing the entire insert (and several hundred base pairs of vector sequences) was measured as $\sim 140 \mathrm{~kb}$ by pulsedfield gel electrophoresis. A 500-bp probe isolated from the +140 $\mathrm{kb}$ end of the insert was able to detect a SacI polymorphism distinguishing castaneus and domesticus mice. This polymorphism cosegregated in 37 of 37 mice with a polymorphism from upstream of the $H 19$ gene, demonstrating genetic linkage of the DNAs from the two ends of the BAC insert, thus indicating that the BAC insert was not chimeric.

\section{H19-BAC1 transgenic mice}

A total of $40 \mu \mathrm{g}$ of H19-BAC1 DNA was digested with NotI to release the mouse DNA insert and electroporated with an equimolar amount of plasmid pMC1neoPolyA (Stratagene) into RI embryonic stem cells. Drug-resistant colonies were selected and screened for the presence of both ends of the BACl insert and for absence of BAC vector sequences. Two of the 44 colonies tested met these criteria. Southern analysis demonstrated that a single copy of the H19-BAC1 was present in each cell line. The cell lines were injected into C57/BL6-J blastocysts to generate chimeric founder mice.

\section{RNA preparation}

Tissues were homogenized in TRIZOL Reagent (Life Technologies) with a power homogenizer (Polytron), and the RNA was isolated according to Life Technologies protocols.

\section{SNuPE}

cDNAs were synthesized with $2 \mu \mathrm{g}$ of DNase I-treated RNA as a template by the Superscript Preamplification System (GIBCO BRL). cDNAs were amplified with $H 19$ or Igf2-specific primers to generate 164- and 574-bp fragments (Srivastava et al. 2000). Purified PCR products were analyzed as described (Srivastava et al. 2000) except that the extension primers used for H19 and Igf2 were 5'-GGCAGCATTGCCAAAGAGG-3' and 5'-GCCAATCAAATTTGGTTTTTTAGAA-3' , respectively, and incorporation of radioactively labeled dCTP and TTP was monitored in each case. Domesticus H19 and castaneus Igf2 templates incorporate TTP, whereas castaneus H19 and domesticus Igf2 templates incorporate dCTP. Minus reverse transcriptase controls were included with each sample. Relative incorporation of the two nucleotides by SNuPE was used to calculate the contribution of the paternal allele to the total H19 RNA pool [T/(C+T)] and the contribution of the maternal allele to the total Igf2 RNA pool $[\mathrm{C} /(\mathrm{C}+\mathrm{T})]$.

\section{In vitro mapping of the $\mathrm{H} 19$ muscle enhancers}

DNA fragments spanning the +10 - to $+42-\mathrm{kb}$ region downstream of $H 19$ were cloned into the multiple cloning site of the reporter vector, pßgal-Promoter (Clontech). This vector carries an SV4O promoter inserted upstream of the $1 a c Z$ gene. Sol8 myoblasts were grown and transfected essentially as described (Calvo et al. 1999), except that for stable transfections, pTK-Hyg (Clontech) was cotransfected with the reporter constructs and resistant colonies were selected for 10 days in $360 \mu \mathrm{g} / \mathrm{ml}$ hygromycin B. Resistant cells were replated and grown $48 \mathrm{hr}$ before switching to differentiation medium containing reduced levels of serum supplements. After 72 additional hr, cell lysates were prepared and $\beta$-galactosidase activity was measured using the Promega Enzyme assay system and was normalized to total protein in the cell extracts. To normalize plasmid uptake for each transient transfection, DNA extracted from $50 \%$ of the transfected cells was digested with BamHI and HindIII and analyzed by Southern blot using a probe specific to the pßgal-Promoter vector. The $\mathrm{C} 2 \mathrm{C} 12$ cell line was grown and transfected by an identical procedure, except that only transient transfection was performed.

\section{In vitro mapping of the insulator}

Reporter plasmid pKP- 1 carries the $H 19$ promoter from -800 to $+6 \mathrm{bp}$ upstream of the Neomycin-resistance gene. A 2.5-kb $X b a \mathrm{I}-X b a \mathrm{I}-\mathrm{Bg} / \mathrm{II}$ fragment carrying the $\mathrm{H} 19$ endodermal enhancers or a 256-bp fragment carrying the SV4O enhancers were cloned upstream of the $H 19$ promoter of pKP-1 to make pKP-2 and pKP-6, respectively. pKP-3 is derived from pKP-2 but carries an $4.7-\mathrm{kb} \mathrm{XbaI-XbaI}$ fragment inserted upstream of the H19 enhancers. This fragment contains $H 19$ sequences from -5.5 to $-0.8 \mathrm{~kb}$. pKP-4 is identical to $\mathrm{pKP}-3$ except that the $\mathrm{XbaI}-\mathrm{XbaI}$ fragment is also inserted between the promoter and enhancer; thus, this $X b a I$ fragment flanks the enhancer. pKP-5 is identical to pKP-4 except that a 2.4-kb BgIII-BgIII fragment was cloned in to flank the enhancer element. pKP-7 is identical to pKP-5 except that the parent plasmid is pKP-6. Thus, the potential insulator element flanks the SV4O enhancer rather than H19 endodermal enhancer sequences. pKP-8 is similar to pKP-7 except that a 2.3-kb BgIII-BamHI fragment from downstream of the $H 19$ gene was inserted to flank the SV4O enhancers.

The human hepatoma cell line, Hep3B, was grown in DMEM with $10 \%$ FBS. Cells were transfected with $10 \mu \mathrm{g}$ of DNA using the Calcium Phosphate Transfection Kit (Invitrogen) according to the manufacturer's instructions. After $72 \mathrm{hr}$, cells were trypsinized and replated at 1:10 dilution in growth medium containing $500 \mu \mathrm{g} / \mathrm{ml} \mathrm{G418.} \mathrm{Resistant} \mathrm{colonies} \mathrm{were} \mathrm{counted} 10$ days after selection.

\section{Acknowledgments}

We thank Shirley Tilghman for $H 19^{\Delta 13}$ mice.

The publication costs of this article were defrayed in part by payment of page charges. This article must therefore be hereby marked "advertisement" in accordance with 18 USC section 1734 solely to indicate this fact.

\section{Note added in proof}

Recent papers have also demonstrated insulator activity for the DMR in vitro and have demonstrated methylation-inhibited binding of the transcriptional regulator, CTCF, to sequences from this region: Bell and Felsenfeld 2000 (Nature 405: 482485), Hark et al. 2000 (Nature 405: 486-489), Kanduri et al. 2000 (Curr. Biol. 10: 449-457), and Szabó et al. 2000 (Curr. Biol. 10: 607-610). 


\section{References}

Ainscough, J.F.-X., Koide, T., Tada, M., Barton, S., and Surani, A. 1997. Imprinting of Igf2 and $H 19$ from a $130 \mathrm{~kb}$ YAC transgene. Development 124: 3621-3632.

Ainscough, J.F.-X., Dandola, L., and Surani, M.A. 2000. Appropriate expression of the mouse $\mathrm{H} 19$ gene utilizes three or more distinct enhancer regions spread over more than 130 kb. Mech. Dev. 91: 365-368.

Bartolomei, M.S., Zemel, S., and Tilghman, S.M. 1991. Parental imprinting of the mouse $H 19$ gene. Nature 351: 153-155.

Bartolomei, M.S., Webber, A.L., Brunkow, M.E., and Tilghman, S.M. 1993. Epigenetic mechanisms underlying the imprinting of the mouse H19 gene. Genes \& Dev. 7: 1663-1673.

Bell, A. and Felsenfeld, G. 1999. Stopped at the border: Boundaries and insulators. Curr. Opin. Genet. Dev. 9: 191-198.

Brandeis, M., Kafri, T., Ariel, M., Chaillet, J.R., McCarrey, J., Razin, A., and Cedar, H. 1993. The ontogeny of allele-specific methylation associated with imprinted genes in the mouse. EMBO J. 12: 3669-3677.

Brenton, J.D., Drewell, R.A., Viville, S., Hilton, K.J., Barton, S.C., Ainscough, J.F.-X., and Surani, M.A. 1999. A silencer element identified in Drosophila is required for imprinting of $H 19$ reporter transgenes in mice. Proc. Natl. Acad. Sci. 96: $9242-9247$.

Brunkow, M.E. and Tilghman, S.M. 1991. Ectopic expression of the $H 19$ gene in mice causes prenatal lethality. Genes \& Dev. 5: 1092-1101.

Cai, H. and Levine, M. 1995. Modulation of enhancer-promoter interactions by insulators in the Drosophila embryo. Nature 376: $533-536$.

Calvo, S., Venepally, P., Cheng, J., and Buonanno, A. 1999. Fiber-type-specific transcription of the troponin I slow gene is regulated by multiple elements. Mol. Cell. Biol. 19: 515-525.

Chung, J.H., Whiteley, M., and Felsenfeld, G. 1993. A 5' element of the chicken $\beta$-globin domain serves as an insulator in human erythroid cells and protects against position effect in Drosophila. Cell 74: 505-514.

Corces, V. and Geyer, P. 1991. Interactions of retrotransposons with the host genome: The case of the gypsy element of Drosophila. Trends Genet. 7: 86-90.

DeChiara, T.M., Robertson, E.J., and Efstratiadis, A. 1991. Parental imprinting of the mouse insulin-like growth factor II gene. Cell 64: 849-859.

Elson, D.A. and Bartolomei, M.S. 1997. A 5' differentially methylated sequence and the 3 '-flanking region are necessary for H19 transgene imprinting. Mol. Cell. Biol. 17: 309-317.

Ferguson-Smith, A.C., Sasaki, H., Cattanach, B.M., and Surani, M.A. 1993. Parental-origin-specific epigenetic modifications of the mouse H19 gene. Nature 362: 751-755.

Geyer, P. and Corces, V.G. 1992. DNA position-specific repression of transcription by a Drosophila zinc-finger protein. Genes \& Dev. 6: 1865-1873.

Gould, T.D. and Pfeifer, K. 1998. Imprinting of mouse Kvlqt1 is developmentally regulated. Hum. Mol. Genet. 7: 483-487.

Hark, A.H. and Tilghman, S.M. 1998. Chromatin conformation of the H19 epigenetic mark. Hum. Mol. Genet. 9: 19791985.

Hebbes, T.R., Clayton, A.L., Thorne, A.W., and Crane-Robinson, C. 1994. Core histone hyperacetylation co-maps with generalized DNase I sensitivity in the chicken beta-globin chromosomal domain. EMBO J. 13: 1823-1830.

Kellum, R. and Schedl, P. 1991. A position-effect assay for boundaries of higher order chromosomal domains. Cell 64: 941-950.

1992. A group of scs elements function as domain boundaries in an enhancer-blocking assay. Mol. Cell. Biol. 12: 2424-2431.

Khosla, S., Aitchison, A., Gregory, R., Allen, N.D., and Feil, R. 1999. Parental allele-specific chromatin configuration in a boundary-imprinting-control element upstream of the mouse H19 gene. Mol. Cell. Biol. 19: 2556-2566.

Kuppuswamy, M.N., Hoffman, J.W., Kasper, C.K., Spitzer, S.G., Groce, S.L., and Bajaj, S.P. 1991. Single nucleotide primer extension to detect genetic diseases: Experimental application to hemophilia B (factor X) and cystic fibrosis. Proc. Nat1. Acad. Sci. 88: 1143-1147.

Lasko, M. 1996. Efficient in vivo manipulation of mouse genomic sequences at the zygote stage. Proc. Natl. Acad. Sci. 93: $5860-5865$.

Leighton, P.A., Ingram, R.S., Eggenschwiler, J., Efstratiadis, A., and Tilghman, S.M. 1995a. Disruption of imprinting caused by deletion of the $H 19$ gene region in mice. Nature 375: 34 39.

Leighton, P.A., Saam, J.R., Ingram, R.S., Stewart, C.L., and Tilghman, S.M. 1995b. An enhancer deletion affects both $H 19$ and Igf2 expression. Genes \& Dev. 9: 2079-2089.

McCarrick, J.W., Parnes, J.R., Seong, R.H., Solter, D., and Knowles, B.B. 1993. Positive-negative selection gene targeting with the diptheria toxin A-chain gene in mouse embryonic stem cells. Transgenic Res. 2: 183-190.

Olek, A. and Walter, J. 1997. The ontogeny of the H19 methylation imprint. Nat. Genet. 17: 275-276.

Pfeifer, K., Leighton, P.A., and Tilghman, S.M. 1996. The structural $\mathrm{H} 19$ gene is required for transgene imprinting. Proc. Natl. Acad. Sci. 93: 13876-13883.

Roseman, R.R., Pirotta, V., and Geyer, P.K. 1993. The su(Hw) protein insulates expression of the Drosophila melanogaster white gene from chromosomal position-effects. EMBO J. 12: 435-442.

Sasaki, H., Jones, P.A., Chaillet, J.R., Ferguson-Smith, A.C., Barton, S.C., Reik, W., and Surani, M.A. 1992. Parental imprinting: Potentially active chromatin of the repressed maternal allele of the mouse Insulin-like Growth Factor II (Igf2) gene. Genes \& Dev. 6: 1843-1856.

Sauer, B. and Henderson, N. 1990. Targeted insertion of exogenous DNA into the eukaryotic genome by the Cre recombinase. New Biol. 2: 441-449.

Schmidt, J., Levorse, J., and Tilghman, S.M. 1999. Enhancer competition between $H 19$ and Igf2 does not mediate their imprinting. Proc. Natl. Acad. Sci. 96: 9733-9738.

Scott, K.S. and Geyer, P.K. 1995. Effects of the su(Hw) insulator protein on the expression of the divergently transcribed Drosophila yolk protein genes. EMBO J. 14: 6258-6267.

Singer-Sam, J., Chapman, V., LeBon, J.M., and Riggs, A.D. 1992. Parental imprinting studies by allele-specific primer extension after PCR: Paternal X chromosome-linked genes are transcribed prior to preferential paternal $\mathrm{X}$ chromosome inactivation. Proc. Natl. Acad. Sci. 89: 10469-10473.

Srivastava, M., Hsieh, S., Grinberg, A., Williams-Simons, L., Huang, S.-P., and Pfeifer, K. 2000. H19 and Igf2 monoallelic expression is regulated in two distinct ways by a shared cis acting regulatory element upstream of H19. Genes \& Dev. 14: 1186-1195.

Sun, F.-L. and Elgin, S.C.R. 1999. Putting boundaries on silence. Cell 99: 459-462.

Szabo, P.A. and Mann, J.R. 1995. Biallelic expression of imprinted genes in the mouse germ line: Implications for erasure, establishment, and mechanisms of genomic imprinting. Genes \& Dev. 9: 1857-1868.

Szabo, P.A., Pfeifer, G.P., and Mann, J.R. 1998. Characterization of novel parent-specific epigenetic modifications upstream 
of the imprinted mouse $H 19$ gene. Mol. Cell. Biol. 18: 67676776.

Thorvaldsen, J.L., Duran, K.L., and Bartolomei, M.S. 1998. Deletion of the $H 19$ differentially methylated domain results in loss of imprinted expression of H19 and Igf2. Genes \& Dev. 12: 3693-3702.

Tremblay, K.D., Saam, J.R., Ingram, R.S., Tilghman, S.M., and Bartolomei, M.S. 1995. A paternal-specific methylation imprint marks the alleles of the mouse H19 gene. Nat. Genet. 9: 407-413.

Tremblay, K.D., Duran, K.L., and Bartolomei, M.S. 1997. A 5' 2-kilobase-pair region of the imprinted mouse $H 19$ gene exhibits exclusive paternal methylation throughout development. Mol. Cell. Biol. 17: 4322-4329.

Udvardy, A., Maine, E., and Schedl, P. 1985. The 87A7 chromomere. Identification of novel chromatin structures flanking the heat shock locus that may define the boundaries of higher order domains. J. Mol. Biol. 185: 341-358.

Webber, A., Ingram, R.I., Levorse, J., and Tilghman, S.M. 1998. Location of enhancers is essential for imprinting of H19 and Igf2. Nature 391: 711-715.

Yoo-Warren, H., Pachnis, V., Ingram, R.S., and Tilghman, S.M. 1988. Two regulatory domains flank the mouse $H 19$ gene. Mol. Cell. Biol. 8: 4707-4715. 


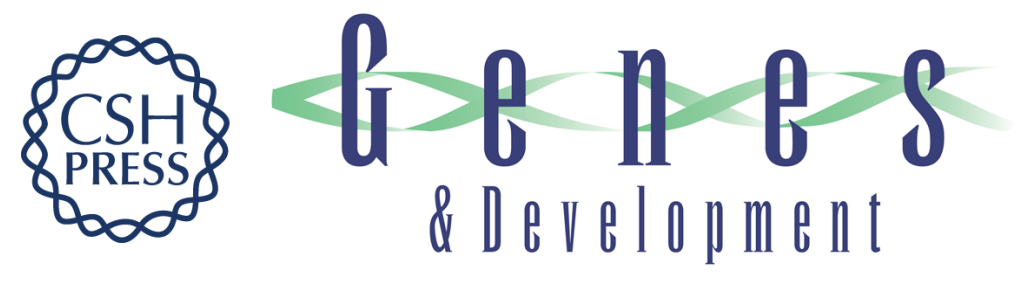

\section{A transcriptional insulator at the imprinted $H 19 /$ lgf2 locus}

Christopher R. Kaffer, Madhulika Srivastava, Kye-Yoon Park, et al.

Genes Dev. 2000, 14:

Access the most recent version at doi:10.1101/gad.14.15.1908

References This article cites 44 articles, 22 of which can be accessed free at: http://genesdev.cshlp.org/content/14/15/1908.full.html\#ref-list-1

License

Email Alerting Receive free email alerts when new articles cite this article - sign up in the box at the top Service right corner of the article or click here.

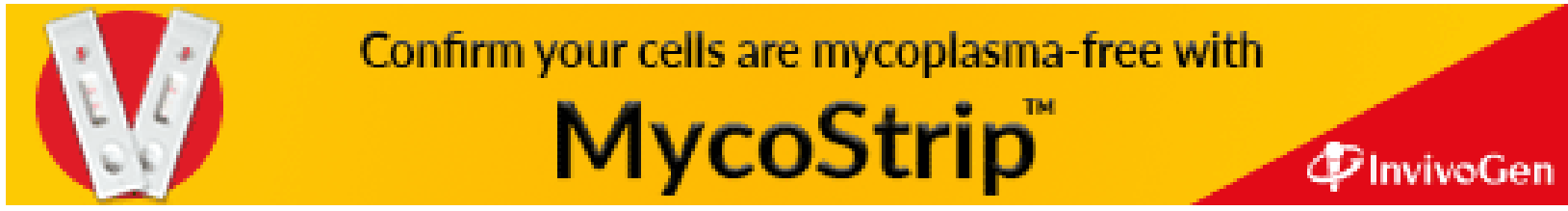

